\title{
Search for Supersymmetry in Gauge Mediated SUSY breaking scenarios at CMS
}

\author{
Christian AUTERMANN* \\ on behalf of the CMS Collaboration \\ I. Phys. Inst. B, RWTH Aachen University \\ E-mail: christian.autermannecern.ch
}

Four CMS searches for Gauge Mediated Supersymmetry in up to $20 \mathrm{fb}^{-1}$ data collected at a center of mass energy of $8 \mathrm{TeV}$ are discussed in this article. Different analysis strategies have been pursued to ensure a good coverage of possible signal scenarios at the best possible sensitivity. Wino-like neutralino mixings are probed in the single-photon final state, while the di-photon final state is most sensitive to bino-like neutralino mixings. Abandoning the large discriminating power of a missing transverse energy requirement opens the sensitivity to a wide range of scenarios but requires novel background estimation techniques developed by the analysis of Stealth Supersymmetry. A fourth analysis uses the multi-lepton final state to search for electroweak produced Supersymmetry. No excess of data over the Standard Model expectation has been observed and upper limits on various signal cross sections were calculated and interpreted in the scenario of General Gauge Mediation.

The European Physical Society Conference on High Energy Physics -EPS-HEP2013 18-24 July 2013

Stockholm, Sweden

* Supported by the German Federal Ministry of Education and Research 
The relevant Standard Model background estimation for inclusive CMS [1] searches in the single- and the di-photon final states $[2,3]$ is extracted purely from data and validated in Monte Carlo simulation. The dominant background from QCD and direct $\gamma$-jet production is modeled using a control sample with loosely isolated photon-like jets, resembling the photons in the signal. The sub-leading EWK background originates from electrons being misidentified as photons. It is modeled using events containing electrons, properly weighted according to the $e \rightarrow \gamma$ misidentification rate measured in data on the $Z \rightarrow e e$ peak. For the single-photon analysis also initial and final state radiation in $W, t \bar{t}$, and $Z$ events is relevant, which is taken from Monte Carlo simulation. The observed data is in good agreement with the Standard Model background-only expectation. Both analyses use several statistically independent high MET bins to calculate CLs limits at 95\% confidence level using a LHC-style treatment of the nuissance parameters. Cross section limits in the General Gauge Mediation (GGM) framework as well as exclusion contours for strong and electro-weak production for bino- and wino-like neutralinos at $7 \mathrm{TeV}$ with $5 \mathrm{fb}^{-1}$ and at $8 \mathrm{TeV}$ with $4 \mathrm{fb}^{-1}$ in dependence of squark, gluino, neutralino, and chargino masses have been calculated. In Fig. $1 \mathrm{a}, \mathrm{b}$ the $8 \mathrm{TeV}$ exclusion contours corresponding to $4 \mathrm{fb}^{-1}$ for wino- and bino-like neutralino production in dependence of the squark- and gluino masses are shown.

The search for Stealth SUSY with little MET [4] makes no use of the large discriminative power of a MET cut and therefore requires new techniques to suppress the Standard Model background, but opens sensitivity to a wide range of scenarios like extra dimensions, heavy-flavor compositeness, little Higgs, R-parity violation, and GGM. Two exclusive signal regions defined by the scalar sum of the transverse momenta of all jets, photons and MET $S_{T}>700 \mathrm{GeV}$ and 4 jets or $\geq 5$ jets are used. Further, two control regions are defined: A ,jet multiplicity sideband" (JMSB) composed of events with two or three jets and $S_{T}>600 \mathrm{GeV}$, and a ", $S_{T}$ sideband" with four or more jets and $600 \mathrm{GeV}<S_{T}<700 \mathrm{GeV}$. The SM expectation is estimated from the data based on the observation that the shape of the ST spectrum of the SM background is independent of jet multiplicity. The $S_{T}$ shape is taken from the JMSB and the normalization is taken from the ST sideband. Limits, calculated in the same way as discussed above, on the stealth SUSY cross section as a function of the squark mass are compared in Fig. 1c to the predicted stealth SUSY cross section. The production of squarks with masses $<1430 \mathrm{GeV}$ can be excluded by this analysis.

The primary non-reducible background of the search for electroweak SUSY production [5] is coming from $\mathrm{ZZ}$ and is estimated using simulation. Additional background from fake and nonprompt leptons arise. These are modeled using data sideband regions with non-isolated leptons. Eight signal regions in exclusive bins of MET between zero and $>200 \mathrm{GeV}$ are defined. The $\mathrm{Z}+$ jets background is suppressed in the high MET bins by the invariant di-lepton and di-jet mass cuts. The dominant background to this search is $t \bar{t}$, which is further reduced by applying a b-tag veto. The remaining $t \bar{t}, \mathrm{WW}$, and single-top background is characterized by an equal rate of $e e+\mu \mu$ versus $e \mu$. This flavor symmetric background is evaluated on a $e \mu$ sample. The remaining non-flavor symmetric background like WZ is taken from simulation and is found to be negligible. Amongst others, a specific gauge-mediated supersymmetry breaking (GMSB) Z-enriched higgsino model is considered, that enhances the ZZ + MET final state. In Fig. 1d the CLs limit obtained by the combination of the four lepton and the two lepton plus two jet analyses on the GMSB cross section versus the mass scale $\mu$, a measure for the degenerate gaugino masses, is shown. The branching fraction to the $\mathrm{ZZ}+\mathrm{ET}$ final state varies from $100 \%$ at $\mu=130 \mathrm{GeV}$ to $85 \%$ at $\mu=410 \mathrm{GeV}$. 

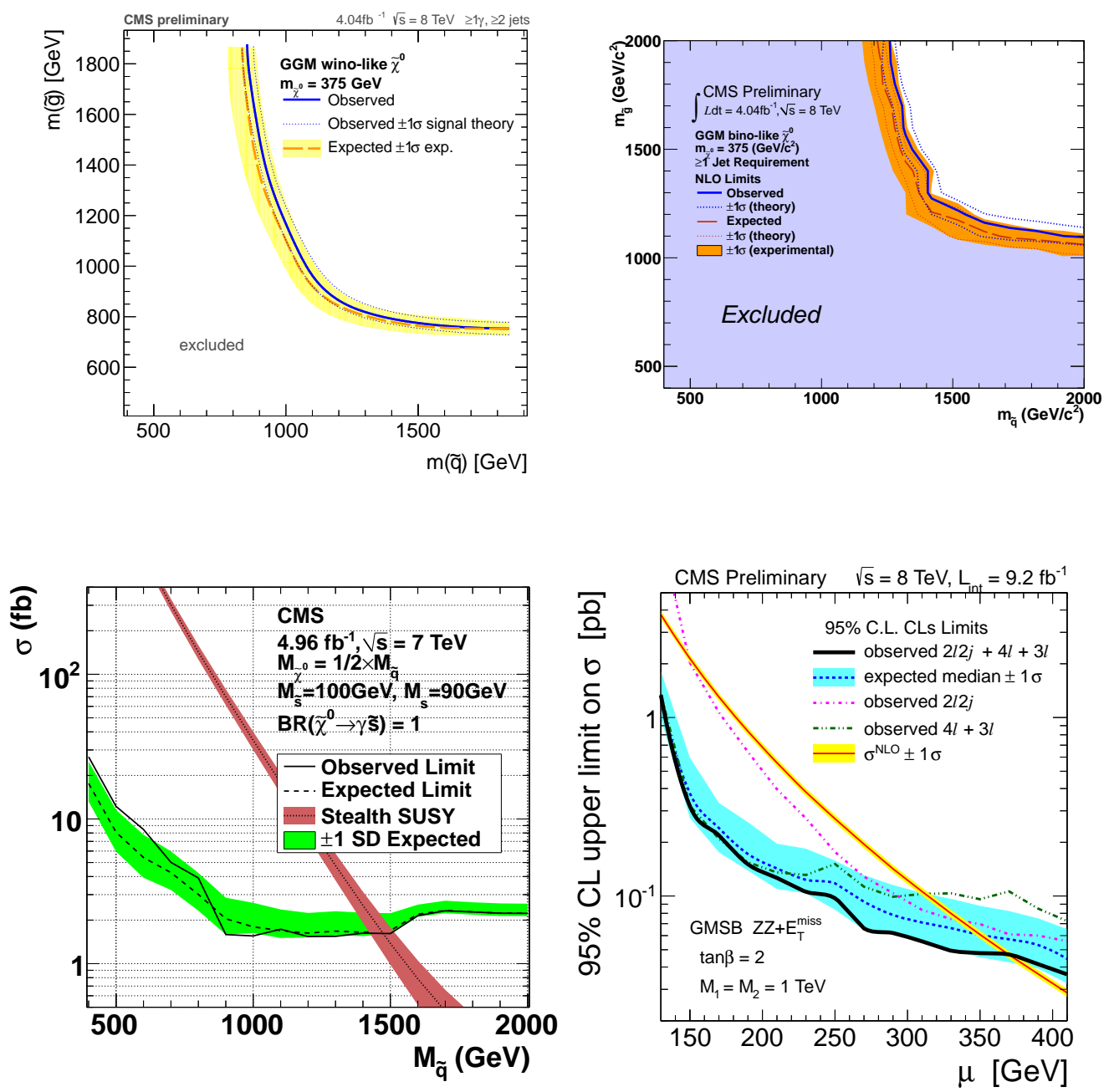

Figure 1: Observed and expected exclusion contours at 95\% CL in the squark mass versus gluino mass plane for the single-photon analysis for wino-like neutralino mixing (top left), the di-photon analysis for bino-like neutralino mixing (top right), the Stealth Analysis limit (bottom left), and the electroweak production limit (bottom right) are shown. The mass scale $\mu$ is a measure for the degenerate gaugino masses.

\section{References}

[1] The CMS Collaboration, ,The CMS experiment at the CERN LHC”, JINST 3:S08004, 2008.

[2] The CMS collaboration „Search for supersymmetry in events with photons and missing energy”, CMS-PAS-SUS-12-018, 2012.

[3] The CMS collaboration, ,Search for new physics in events with photons, jets, and missing transverse energy in pp collisions at $\sqrt{s}=7$ TeV”, J. High Energy Phys. 03 (2013) 111.

[4] The CMS collaboration ,Search for supersymmetry in events with photons and low missing transverse energy in pp collisions at $\sqrt{s}=7$ TeV", Phys. Lett. B 719 (2013) 42.

[5] The CMS collaboration „Search for direct EWK production of SUSY particles in multilepton modes with 8 TeV data", CMS-PAS-SUS-12-022, 2012. 